\title{
Efeito agudo da marcha em esteira com estímulo auditivo sobre parâmetros cinemáticos da marcha e mobilidade em Parkinsonianos
}

\author{
Acute effect of treadmill training with auditory stimulation on gait \\ kinematic parameters and mobility in Parkinsonians
}

Patrícia de Aguiar Yamada1 ${ }^{*}$, Késia Maísa Amaral-Felipe ${ }^{1}$, Bárbara Fernandes Rodrigues ${ }^{2}$,
Maira Peloggia Cursino ${ }^{1}$, Camilla Zamfolini Hallal ${ }^{3}$, Flávia Roberta Faganello-Navega ${ }^{2}$
ARTIGO ORIGINAL | ORIGINALARTICLE

RESUMO

O objetivo do presente estudo foi verificar o efeito agudo da marcha em esteira com estímulo auditivo sobre parâmetros cinemáticos da marcha e mobilidade em Parkinsonianos. Participaram 14 indivíduos com Parkinsonismo idiopático, os quais foram divididos em 2 grupos: grupo intervenção e grupo controle. Foi realizada avaliação, composta por dois testes que avaliam a mobilidade funcional, nomeados Short Physical Performance Battery (SPPB) e Timed up and go (TUG); avaliação da velocidade de marcha através do teste de 10 metros e análise do comprimento de passo em um circuito de 16 metros, em seguida os participantes foram submetidos a uma sessão de marcha em esteira, sendo o grupo intervenção com auxílio de estímulos sonoros e o grupo controle sem estímulos. Ao final da intervenção os indivíduos foram reavaliados. Os resultados mostraram que ambos os grupos apresentaram melhora da mobilidade no teste TUG $(p=0.003)$, da velocidade de marcha no teste de 10 metros $(p=0.004)$ e do comprimento de passo $(p=0.029)$, porém não houve diferença estatística entre os grupos. O SPPB não apresentou diferença significativa após o protocolo de marcha $(p=0.118)$. Conclui-se que uma sessão de marcha em esteira foi efetiva na melhoria dos parâmetros cinemáticos da marcha e mobilidade em estudo, porém a associação do estímulo auditivo não influenciou as variáveis analisadas.

Palavras-chave: doença de Parkinson, marcha, esteira ergométrica, estímulo auditivo

ABSTRACT

The aim of this study was to investigate the acute effect of treadmill training with auditory stimulation on gait kinematic parameters and mobility in Parkinsonians. Participants were 14 individuals with idiopathic Parkinsonism, which were divided into 2 groups: intervention and control group. All patients were assessed through two tests that assess functional mobility, named Short Physical Performance Battery (SPPB) and Timed up and go (TUG); gait speed evaluation by the 10 meters test; and analysis of the step length on a 16-meter circuit, then participants were subjected to a treadmill gait session, intervention group with sound stimuli support and control group without stimuli. At the end of the intervention subjects were reassessed. The results showed that both groups improved mobility in the TUG test $(p=0.003)$, gait speed in the 10 meters test $(p=0.004)$ and step length $(p=0.029)$, but there was no statistical difference between groups. The SPPB did not change significantly after the gait protocol $(p=0.118)$. It is concluded that one treadmill gait session was effective in improving the gait kinematic parameters and mobility study, but the combination of auditory stimuli did not significantly changed the analyzed variables.

Keywords: Parkinson's disease, gait, treadmill, auditory stimulus.

Artigo recebido a 30.12.2015; Aceite a 28.05.2016

${ }^{1}$ Universidade Estadual Paulista, UNESP, Rio Claro, SP, Brasil

${ }^{2}$ Universidade Estadual Paulista, UNESP, Marília, SP, Brasil

${ }^{3}$ Universidade Federal de Uberlândia, UFU, Uberlândia, MG, Brasil

* Autor correspondente: Universidade Estadual Paulista, Departamento de Fisioterapia e Terapia Ocupacional, Avenida Hygino Muzzi Filho, 737, CEP 17525-000, Marília, SP, Brasil. E-mail: pathy_yamada@hotmail.com 


\section{INTRODUÇ̃̃o}

A doença de Parkinson (DP) é uma patologia do sistema nervoso central de caráter progressivo que envolve os gânglios da base, causando a degeneração das células dopaminérgicas que formam a via nigro-estriatal, o que resulta na inibição do movimento (Mehndiratta, Garg, \& Pandey, 2011). A doença acomete um em cada mil indivíduos, porém a prevalência aumenta com a idade, afetando geralmente indivíduos acima dos 50 anos (Morris, 2000). Dentre os principais prejuízos motores pode-se citar o tremor de repouso, a rigidez, bradicinesia, alterações posturais e da marcha (Kummer, Cardoso, \& Teixeira, 2010), sendo a ultima, um dos principais limitantes da autonomia e qualidade de vida dos pacientes com DP, uma vez que constituem a principal causa de quedas, inatividade e isolamento social (Scalzo, Flores, Marques, Robini, \& Teixeira, 2012).

A marcha apresenta grandes mudanças temporal e espacial, como encurtamento do comprimento de passo (CP) e largura de passo (LP), diminuição da elevação dos pés com consequente arrasto, diminuição da velocidade de marcha (VM) ou aumento da mesma, a fim de aumentar o ritmo para compensar a falta de equilíbrio (Seco-Calvo, Gago-Fernández, Canode-la-Cuerda, \& Fernández-de-las-Peñas, 2012).

A realização de programas de reabilitação que combinem atividades motoras e estímulos sensoriais, como o estímulo auditivo (EA) externo, vem sendo cada vez mais destacada na literatura (Fernandez-del-Olmo, Arias, \& Cudeiro-Mazaira, 2004). Segundo Nieuwboer, Rochester, e Jones (2008), a utilização de pistas auditivas externas é capaz de tornar as áreas parietal lateral, tálamo e áreas pré-motoras mais envolvidas na realização do movimento, proporcionando um aumento da excitabilidade dos neurônios motores através da via retículoespinal, desviando dos circuitos do núcleo da base, deficiente em parkinsonianos, os quais normalmente teriam sua atividade aumentada no início de um movimento, quando sem o auxílio de pistas externas. Dessa forma, indivíduos com DP tornam-se capazes de gerar padrões adequados de marcha, melhorando variáveis como CP, cadência e VM (Aminian, Najafi, Büla, Leyvraz, \& Robert, 2002).

Outra ferramenta utilizada na melhora da marcha em indivíduos com DP é a esteira ergométrica. Segundo Bello, Marquez, Camblor, e Fernandez-Del-Olmo (2010), o efeito terapêutico da esteira pode estar associado ao estímulo sensorial proprioceptivo oferecido pelo movimento da faixa da esteira, capaz de melhorar os padrões da marcha destes pacientes. Segundo os autores, o treino é capaz de melhorar a VM, a LP e o CP em indivíduos em estágios médio a moderado da DP. Além disso, os autores sugerem que uma das vantagens do treino em esteira é o fato de ela ser acessível na maioria das clínicas de fisioterapia.

Estudos têm demonstrado que uma única sessão de treinamento de marcha em esteira é uma intervenção eficiente para melhorar parâmetros da marcha em indivíduos com DP (Bello, Sanchez, \& Fernandez-del-Olmo, 2008; Frenkel-Toledo et al., 2005), assim como o EA externo também tem mostrado efeito benéfico imediato sobre o padrão de marcha em solo de parkinsonianos (Almeida et al., 2015; Matsumoto, Magalhães, Antunes, \& TorrianiPasin, 2014). Nesse sentido, é de fundamental importância verificar se o treino de marcha em esteira associado ao EA é capaz de proporcionar maiores alterações nas características cinemáticas da marcha de quando o treino é realizado sem EA e em solo. Tais resultados poderão servir de base para que os profissionais fisioterapeutas empreguem esse tipo de treinamento em seus pacientes, pois mesmo sendo de conhecimento que o treino de marcha com EA é benéfico, este ainda é de difícil aplicação devido ao espaço restrito da maioria das clínicas. Se as alterações positivas de fato ocorrem e por ser a esteira ergométrica um equipamento presente na maioria das clínicas, este estudo contribuirá para que um novo tipo de treino se torne comum na prática clínica para melhora do padrão de macha do indivíduo com DP e consequente melhoria de sua mobilidade e qualidade de vida.

Assim, o presente estudo teve por objetivo verificar o efeito agudo da marcha em esteira com 
EA sobre parâmetros cinemáticos da marcha e mobilidade em Parkinsonianos.

Nós hipotetizamos que, com uma única sessão de marcha em esteira associada à EA em indivíduos com DP, ocorra melhora dos parâmetros cinemáticos da marcha destes indivíduos, reduzindo assim o risco de quedas e podendo aumentar sua mobilidade funcional.

\section{MÉTODO}

Trata-se de um ensaio clínico randomizado controlado, o qual foi aprovado pelo Comitê de Ética em Pesquisa da Faculdade de Filosofia e Ciências da UNESP - Marília (nº 0995/2014). Todos os voluntários foram informados sobre os procedimentos da pesquisa e foram esclarecidos também quanto ao sigilo das informações coletadas e das identidades dos mesmos. Após terem lido e concordado, assinaram o Termo de Consentimento Livre e Esclarecido.

\section{Participantes}

Participaram do estudo 14 indivíduos do gênero masculino $(n=9)$ e feminino $(n=5)$, com diagnóstico de Parkinsonismo idiopático, classificados nos estágios de 1 a 3 (incapacidade leve a moderada) da escala de Hoehn e Yahr (Hoehn \& Yahr, 1967), nos quais é possível a realização de marcha independente. Os indivíduos foram recrutados no período de Março a Agosto de 2014, a partir de divulgação por meio televisivo, panfletagem em terminal de ônibus, consultórios médicos, hospitais, unidades básicas de saúde, além de consulta em prontuários médicos no hospital de referência da cidade de Marília/SP e indicação fisioterapêutica dos serviços da cidade. Os pesquisadores realizaram um levantamento sobre o histórico clínico dos participantes, selecionando apenas os indivíduos com diagnóstico médico de DP.

Os critérios de elegibilidade incluíam a realização de marcha independente sem o uso de dispositivos auxiliares, não estar em fase de adaptação farmacológica, realizar as avaliações e intervenções na fase "on" dos medicamentos para DP, não apresentar dor, fratura, ou lesão grave em tecidos moles nos seis meses pregressos ao estudo, bem como histórico de alterações cognitivas, cardiovasculares ou respiratórias não tratadas e ser capaz de compreender as instruções verbais.

Os participantes do estudo foram divididos aleatoriamente em dois grupos: grupo intervenção (GI, $n=7$ ) e grupo controle (GC, $\mathrm{n}=7$ ), sendo a randomização realizada em blocos de sete participantes. Cada participante recebeu um envelope opaco fechado, ao abrir o envelope, caso o número contido fosse par, o participante era direcionado ao grupo controle, caso o número fosse ímpar, este era direcionado ao grupo intervenção.

\section{Instrumentos}

No presente estudo foram avaliados a mobilidade funcional dos participantes através dos testes Short Physical Performance Battery (SPPB) e Timed up and GO (TUG), além das variáveis cinemáticas $\mathrm{VM}$ e $\mathrm{CP}$ através do teste de velocidade de marcha e análise cinemática da marcha em um circuito oval de 16 metros ( $4.8 \mathrm{x}$ 3.2 metros), respectivamente. Durante a coleta de dados cinemáticos no circuito oval foram utilizados também uma câmera Sony ${ }^{\circledR}$ com frequência de amostragem de $60 \mathrm{~Hz}$ e marcadores fotorreflexivos, os quais eram fixados nos seguintes pontos anatômicos do membro inferior direito dos participantes: trocânter maior, face lateral da linha articular do joelho, maléolo lateral, base do segundo dedo de ambos os pés e maléolo medial do membro inferior contralateral. Antes do início da coleta de dados cinemáticos da marcha em solo, foi realizada a calibração do ambiente.

O SPPB é um teste rápido, de baixo custo, passível de ser aplicado por uma só pessoa em espaço físico reduzido e vem sendo amplamente utilizado para avaliação da mobilidade, consiste em três avaliações: equilíbrio estático em três posições (pés unidos, semi-tandem e tandem); $\mathrm{VM}$, cronometrando o tempo gasto para percorrer quatro metros em ritmo normal; e força muscular dos membros inferiores, cronometrando o tempo gasto para levantar-se e sentar-se em uma cadeira por cinco vezes consecutivas sem o auxílio das mãos. O escore total é obtido pela soma das pontuações de cada teste, podendo variar entre 0 a 12 pontos, sendo o desempenho representado através da seguinte 
graduação: 0 a 3 pontos, incapaz ou apresenta desempenho ruim; 4 a 6 pontos, baixo desempenho; 7 a 9 pontos, moderado desempenho e 10 a 12 pontos, bom desempenho (Guralnik et al., 1994).

O TUG é um instrumento importante para avaliar o equilíbrio e mobilidade, nele é registrado o tempo para se levantar de uma cadeira, deambular por uma distância de 3 metros e retornar à cadeira. Maiores valores de tempo representam maior risco de quedas (Podsiadlo \& Richardson, 1991).

O teste de velocidade da marcha é composto de 10 metros e tem como finalidade avaliar componentes temporais da marcha. É requisitado ao paciente que ele caminhe por uma distância de 10 metros em linha reta em sua velocidade de caminhada normal enquanto o tempo é cronometrado. O teste é realizado por três vezes e então se calcula o resultado através da média dos valores obtidos durante as três tentativas. Para eliminar o componente de aceleração e desaceleração, os quais podem influenciar nos valores, são desconsiderados os primeiros e últimos 1.2 metros do percurso (Watson, 2002).

A avaliação cinemática da marcha foi realizada em um circuito oval de 16 metros (4.8 $\times 3.2$ metros) para garantir uma marcha contínua, sem interrupções. Estudos apontam que alterações nos parâmetros da marcha são mais facilmente identificadas em situações de marcha contínua do que em marcha realizada em espaço restrito, pois esta última causa alterações no ritmo locomotor temporal (Kang \& Dingwell, 2008; Paterson, Lythgo, \& Hill, 2009). Além disto, existe uma dependência entre os ciclos da marcha, ou seja, a maneira com que o indivíduo executa um passo é afetada pelos passos anteriores e, deste modo, contínuas interrupções durante a avaliação da marcha podem trazer efeitos adversos na análise do padrão de marcha (Dingwell, Cusumano, Cavanagh, \& Sternad, 2001; Paterson et al., 2009).

Durante a sessão de intervenção, utilizou-se uma esteira ergométrica (Modelo Máster Inbramed $\left.{ }^{\circledR}\right)$ com o auxílio de um cinto de segurança conectado a um cabo de aço fixado a uma estrutura metálica de suporte para evitar quedas; e um metrônomo (Tempo Perfect
Metronome Software), responsável por fornecer pistas auditivas aos participantes.

\section{Procedimentos}

Os procedimentos de coleta de dados foram realizados em dois dias consecutivos, no mesmo horário e de forma individual. No primeiro dia o participante foi orientado detalhadamente sobre os objetivos e procedimentos do estudo, sendo, em seguida, verificada a pressão arterial. A coleta de dados foi iniciada pela avaliação da mobilidade funcional, por meio dos testes Short Physical Performance Battery (SPPB) e Timed up and GO (TUG), depois foi realizada a análise da VM e após, avaliação cinemática da marcha em solo, na qual era solicitado que o indivíduo caminhasse em velocidade de preferência por cinco vezes consecutivas no circuito oval. Ainda no primeiro dia, foi realizada a seleção da velocidade de preferência da marcha em esteira e o protocolo de familiarização proposto por Bello, Sanchez, e Fernandez-del-Olmo (2008), com duração de 20 minutos.

Para a seleção da velocidade de preferência da marcha em esteira o voluntário foi inicialmente submetido a uma velocidade $50 \%$ inferior que a velocidade do Teste de 10 metros. Após compreender o funcionamento da esteira, a velocidade foi aumentada até que o participante referisse estar mais rápido que o usual, assim esse valor foi anotado. Posteriormente, a velocidade foi diminuída até que fosse referido estar mais devagar que a VM do dia-a-dia e novamente o valor foi anotado. Após realizar três vezes esse procedimento, foi realizada a média aritmética das velocidades referidas e assim foi definida a velocidade de preferência para familiarização e treino.

Logo após o protocolo de intervenção (sessão de marcha), o participante foi submetido à reavaliação através dos mesmos métodos acima citados.

Para a análise do $\mathrm{CP}$ foi realizada a normalização pelo comprimento do membro inferior direito de cada voluntário e, em seguida, utilizado o programa Peak Motus Motion Measurement System, versão 9.0, que permite registrar o posicionamento e deslocamento dos segmentos corporais, a partir da marcação dos 
pontos articulares de interesse e controlar a aquisição, a leitura, a digitalização, o tratamento e o armazenamento dos parâmetros cinemáticos para sincronização e posterior análise dos dados.

\section{Sessão de marcha}

No segundo dia, durante a visita experimental, o indivíduo foi submetido ao protocolo de marcha em esteira ergométrica, o qual tinha 30 minutos de duração.

O GI realizou a intervenção em esteira associada ao EA advindo do metrônomo ajustado a uma frequência $20 \%$ menor que a frequência de passo média da marcha em esteira. Este estímulo foi usado para induzir o aumento do $\mathrm{CP}$ por meio da diminuição da cadência da marcha em velocidade constante.

O GC realizou a intervenção em esteira sem associação de qualquer outro tipo de estímulo.

\section{Análise estatística}

Para os dados referentes à caracterização da amostra foram aplicados os testes estatísticos qui-quadrado para dados categóricos e MannWhitney para dados não categóricos.
A normalidade dos dados foi confirmada pelo teste Shapiro-Wilk. Em seguida foi realizado o teste estatístico ANOVA Medidas Repetidas Two Way por meio do software PASW statistics $18.0{ }^{\circledR}$ (SPSS). Em todos os testes estatísticos foi adotado o nível de significância de $p<0.05$.

\section{RESULTADOS}

A caracterização dos sujeitos do estudo está expressa na Tabela 1. A análise estatística não apontou diferenças entre os grupos em todas as variáveis $(\mathrm{p}>0.05)$.

Os resultados da ANOVA Medidas Repetidas Two Way apontaram que não houve interação entre os grupos e o tempo (avaliações pré e pós sessão) em nenhuma das variáveis em estudo ( $p>0.05)$. Os resultados mostraram que ambos os grupos melhoraram significativamente a performance nos testes TUG e VM, e na variável CP. Ainda verificando-se um efeito geral significativo do grupo apenas no CP $(p=0.019)$, ou seja, os valores do CP (considerando simultaneamente os dois momentos de avaliação) no GI foram superiores ao GC. Não se observaram alterações significativas no SPPB (Tabela 2).

Tabela 1

Caracterização da amostra. Valores apresentados em média \pm desvio padrão

\begin{tabular}{cccccc}
\hline & Idade (anos) & Tempo da Doença (anos) & Sexo F/M & Hipertensão (S/N) & Diabetes (S/N) \\
\hline GC & $75.43 \pm 10.10$ & $4.57 \pm 2.23$ & $2 / 5$ & $5 / 2$ & $2 / 5$ \\
GI & $67.14 \pm 6.96$ & $4 \pm 2.58$ & $3 / 4$ & $5 / 2$ & $0 / 7$ \\
\hline
\end{tabular}

$\overline{\mathrm{GC}}=$ Grupo controle; GI = Grupo intervenção; $\mathrm{F} / \mathrm{M}=$ Feminino/Masculino; $\mathrm{S} / \mathrm{N}=$ sim/não. $\mathrm{p}>0.05$.

Tabela 2

Anova Medidas Repetidas, valores de médias e desvios padrão para o conjunto de variáveis analisadas antes e após a intervenção dos grupos GC e GI.

\begin{tabular}{|c|c|c|c|c|c|c|c|c|c|}
\hline & \multirow[b]{4}{*}{ Antes } & \multirow{2}{*}{\multicolumn{2}{|c|}{ GC }} & \multirow{2}{*}{\multicolumn{3}{|c|}{ GI }} & \multicolumn{3}{|r|}{ Grupos } \\
\hline & & & & & & & & & $x$ \\
\hline & & & & & & & Grupos & Avaliação & Avaliação \\
\hline & & Depois & $d$ & Antes & Depois & $d$ & $p$ & $p$ & $p$ \\
\hline SPPB & $8.29 \pm 2.87$ & $9.14 \pm 2.27$ & 0.32 & $9.86 \pm 1.46$ & $10.43 \pm 0.98$ & 0.45 & 0.229 & 0.118 & 0.797 \\
\hline TUG (s) & $18.77 \pm 9.14$ & $17.37 \pm 7.56$ & 0.16 & $12.17 \pm 1.71$ & $11.54 \pm 1.67$ & 0.37 & 0.104 & 0.003 & 0.660 \\
\hline $\mathrm{VM}(\mathrm{m} / \mathrm{s})$ & $0.86 \pm 1.39$ & $0.95 \pm 1.39$ & 0.06 & $1.14 \pm 0.75$ & $1.22 \pm 0.74$ & 0.10 & 0.166 & 0.004 & 0.833 \\
\hline $\mathrm{CP}(\mathrm{cm})$ & $50.50 \pm 21.22$ & $54.09 \pm 16.26$ & 0.18 & $67.80 \pm 6.48$ & $70.15 \pm 0.13$ & 0.31 & 0.019 & 0.029 & 0.683 \\
\hline
\end{tabular}

$\mathrm{VM}=$ velocidade de marcha; $\mathrm{CP}=$ comprimento de passo; $\mathrm{d}=$ tamanho do efeito.

\section{DISCUSSÃO}

O presente estudo teve por objetivo analisar o efeito agudo da marcha em esteira associada ao EA sobre parâmetros cinemáticos da marcha e mobilidade funcional de indivíduos com DP.
Nossos resultados mostraram que ambos os grupos (marcha em esteira e marcha em esteira associada ao EA) obtiveram melhora significativa da mobilidade funcional no teste TUG e dos parâmetros cinemáticos VM e $\mathrm{CP}$, o que sugere 
que a melhora ocorreu devido ao treino de marcha em esteira e que o EA não exerceu influência nos resultados.

A melhora em relação ao teste TUG e às variáveis cinemáticas VM e $\mathrm{CP}$ dos dois grupos submetidos ao treino de marcha em esteira pode ser atribuída ao efeito terapêutico da esteira gerado pelo estímulo sensorial proprioceptivo oferecido pelo movimento da faixa da esteira. Segundo Herman, Giladi, e Hausdorff (2009), a esteira atua como pista externa estimulando receptores proprioceptivos e vestibulares, que por sua vez geram impulsos sensoriais repetitivos para o Sistema Nervoso Central, o que compensa o déficit de pistas internas a respeito do movimento e ritmo da marcha, causado pelos prejuízos dos impulsos internos dos gânglios da base nessa população.

A redução do tempo para a realização do teste TUG pode estar relacionada com a melhora da VM, melhora esta que já foi evidenciada em outros estudos que realizaram treino agudo ou crônico de marcha em esteira sem associação de outros estímulos, demonstrando uma íntima ligação entre tal variável temporal e a mobilidade (Frenkel-Toledo et al., 2005; Talia Herman, Giladi, Gruendlinger, \& Hausdorff, 2007).

A melhora da VM pode ser explicada pela ritmicidade imposta pela esteira, uma vez que diferente da marcha em solo, é necessário manter o ritmo de passo de acordo com a velocidade da esteira, promovendo ciclos rítmicos de marcha. Durante o treino de marcha em ritmo constante, receptores de carga, fusos musculares, órgãos tendinosos de Golgi e outras informações sensoriais são ativados e transferidos para circuitos do sistema neuronal, podendo ocorrer o reforço e a plasticidade neuronal, facilitando desta forma o ritmo da marcha e podendo o mesmo ser mantido nos circuitos neuronais durante o treino e após longos períodos, o que vai de encontro com nossos resultados que evidenciam aumento da VM após o treino agudo de marcha em esteira (Frenkel-Toledo et al., 2005; Toole, Maitland, Warren, Hubmann, \& Panton, 2005).

A manutenção da velocidade constante imposta pela ritmicidade da esteira com ou sem o auxílio de EA induz o sujeito a aumentar o CP, o que sugere que esta funciona como uma pista externa, proporcionando uma estimulação sensorial adequada e que gera em indivíduos com DP um padrão de passo normal, pois, apesar dos déficits pálido corticais encontrados nestes pacientes, o córtex pré motor encontra-se intacto e quando ativado controla externamente os movimentos (Rochester et al., 2005). Além disso, durante a marcha em esteira, enquanto o membro inferior é levado para trás, ocorre o alongamento dos flexores de quadril, provocando uma transmissão de impulsos aos Geradores Centrais de Padrão, os quais são responsáveis por um padrão de marcha mais regular através do aumento do CP (Bello \& Fernandez-Del-Olmo, 2012), fato este que corrobora com os achados do presente estudo e do estudo realizado por Bello et al. (2008), os quais encontraram melhora da cadência, aumento do $\mathrm{CP}$, bem como o aumento da VM em indivíduos com DP, após serem submetidos a uma única sessão de treino de marcha em esteira com duração de 20 minutos.

$O$ fato de não termos encontrado diferença significativa no teste SPPB pode ser justificado pelo fato de este resultar da combinação de vários testes (equilíbrio, VM e força de membros inferiores) e particularmente a componente de força é dificilmente influenciada pelo treino agudo de marcha em esteira. Resultado semelhante foi encontrado por Toole, Maitland, Warren, Hubmann e Panton (2005), em um estudo com 23 indivíduos com DP submetidos a treino de marcha em esteira. Os autores observaram melhora significativa quanto ao padrão de marcha e equilíbrio, porém, a força muscular não sofreu alterações.

Apesar de alguns estudos mostrarem que o EA externo possui efeito imediato na melhora do padrão de marcha de indivíduos com DP (Almeida et al., 2015; Matsumoto et al., 2014), em nosso estudo não houve influência significativa do mesmo sobre os resultados. Entretanto, é importante ressaltar que nos estudos realizados anteriormente, o treino de marcha associado ao EA foi realizado no solo e, em nosso estudo, o treino foi realizado na esteira.

$\mathrm{O}$ fato do EA não ter influenciado em nossos resultados poderá ser explicado pela imposição de duas pistas externas diferentes (o indivíduo 
dirige simultaneamente a sua atenção à esteira e ao EA), configurando uma dupla tarefa. Estudos anteriores já comprovaram a incapacidade de pacientes com DP em realizar atividades de dupla tarefa, devido à falha no controle dos movimentos automáticos (Holtzman \& Gazzaniga, 1982), sendo assim, o déficit no controle automático dos movimentos associado à deficiência da atenção ou memória operacional pode levar esses pacientes à incapacidade de realizar atividades de dupla tarefa.

Um estudo realizado por Suteerawattananon, Morris, Etnyre, Jankovic, e Protas (2004), com 24 indivíduos com DP submetidos a uma única sessão de marcha em solo associada a estímulo auditivo e visual, mostrou melhora da cadência quando submetidos ao EA e melhora do $\mathrm{CP}$ quando submetidos ao estímulo visual, porém o uso simultâneo de ambos os estímulos não resultou em melhora significativa da marcha quando comparado à aplicação individual de cada estímulo. Os autores sugeriram que a associação dos dois estímulos, visual e auditivo, pode ter levado o indivíduo a dividir a sua atenção, prejudicando a melhora da marcha. De maneira semelhante, em nosso estudo, a adição de mais de uma pista externa (esteira mais EA) pode ter interferido na efetividade da realização da marcha. O prejuízo causado pela associação de dois estímulos pode ser devido ao fato de que após a automatização o movimento passa a ocorrer na área do circuito dos núcleos da base (O’Shea, Morris, \& Iansek, 2002), área acometida na DP, tornando a atividade primária, no caso a marcha, prejudicada. $\mathrm{O}$ aumento de erros durante a tarefa primária ou do tempo para a sua execução pode ser chamada de interferência de dupla tarefa, a qual ocorre devido à competição por demandas similares para o processamento entre as duas tarefas (marcha em esteira e EA) (Wu \& Hallett, 2008).

Entre as limitações de nosso estudo estão o número pequeno de participantes, a diferença significativa entre os grupos para o $\mathrm{CP}$, o que pode ter influenciado em nossos resultados, e o fato de termos analisado apenas $\mathrm{o} C \mathrm{CP}$ e não outras variáveis espaciais a fim de tornar a análise mais abrangente. A avaliação da variabilidade e a LP devem ser incluídas em estudos futuros, sendo que a variabilidade mede falhas nos mecanismos neurais de regulação da marcha e o seu aumento indica redução de estabilidade, tornando os indivíduos mais predispostos a quedas; e o aumento da LP indica redução de equilíbrio durante a marcha, resultante de uma instabilidade médio-lateral.

Novos estudos devem ser realizados com uma maior população, uma vez que uma única sessão de intervenção evidenciou benefícios sobre os parâmetros cinemáticos da marcha e mobilidade funcional de indivíduos com DP. Os resultados do presente estudo contribuem para a prática clínica, pois a associação da pista auditiva ao treino de marcha em esteira promoveu melhora nos parâmetros avaliados assim como o treino de marcha em esteira de forma isolada, podendo ambos ser utilizados como forma de tratamento em pacientes com DP e facilitando sua aplicação devido a necessidade de um espaço reduzido para a esteira ergométrica e o fato de esta ser acessível na maioria das clínicas de fisioterapia.

\section{CONCLUSÃO}

Podemos concluir que o treino agudo da marcha em esteira acarretou melhora da mobilidade no teste TUG e dos parâmetros cinemáticos VM e CP em indivíduos com DP, porém a associação do EA ao treino da marcha em esteira não influenciou as variáveis analisadas.

\section{Agradecimentos: \\ Nada a declarar}

\section{Conflito de Interesses:}

Nada a declarar.

\section{Financiamento:}

Nada a declarar

\section{REFERÊNCIAS}

Almeida, I. A. de, Nascimento, T. S. do, Lemes, L. B., Batistetti, C. L., Ferraz, H. B., \& Santos, S. M. S. (2015). Efeito Imediato da Fisioterapia na Marcha em Indivíduos com Doença de Parkinson. Saúde e Pesquisa, 8(2), 247-253. http://doi.org/10.17765/19831870.2015v8n2p247-253 
Aminian, K., Najafi, B., Büla, C., Leyvraz, P.-F., \& Robert, P. (2002). Spatio-temporal parameters of gait measured by an ambulatory system using miniature gyroscopes. Journal of Biomechanics, 35(5), 689-699.

Bello, O., \& Fernandez-Del-Olmo, M. (2012). How does the treadmill affect gait in Parkinson's disease? Current Aging Science, 5(1), 28-34.

Bello, O., Marquez, G., Camblor, M., \& FernandezDel-Olmo, M. (2010). Mechanisms involved in treadmill walking improvements in Parkinson's disease. Gait \& Posture, 32(1), 118-123. http://doi.org/10.1016/j.gaitpost.2010.04.015

Bello, O., Sanchez, J. A., \& Fernandez-del-Olmo, M. (2008). Treadmill walking in Parkinson's disease patients: adaptation and generalization effect. Movement Disorders: Official Journal of the Movement Disorder Society, 23(9), 1243-1249. http://doi.org/10.1002/mds.22069

Dingwell, J. B., Cusumano, J. P., Cavanagh, P. R., \& Sternad, D. (2001). Local dynamic stability versus kinematic variability of continuous overground and treadmill walking. Journal of Biomechanical Engineering, 123(1), 27-32.

Fernandez-del-Olmo, M., Arias, P., \& CudeiroMazaira, F. J. (2004). Facilitación de la actividad motora por estímulos sensoriales en la enfermedad de Parkinson. Revista de Neurologia, 39(9), 841-847.

Frenkel-Toledo, S., Giladi, N., Peretz, C., Herman, T., Gruendlinger, L., \& Hausdorff, J. M. (2005). Treadmill walking as an external pacemaker to improve gait rhythm and stability in Parkinson's disease. Movement Disorders: Official Journal of the Movement Disorder Society, 20(9), 1109_ 1114. http://doi.org/10.1002/mds.20507

Guralnik, J. M., Simonsick, E. M., Ferrucci, L., Glynn, R. J., Berkman, L. F., Blazer, D. G., ... Wallace, R. B. (1994). A short physical performance battery assessing lower extremity function: association with self-reported disability and prediction of mortality and nursing home admission. Journal of Gerontology, 49(2), M85-94.

Herman, T., Giladi, N., Gruendlinger, L., \& Hausdorff, J. M. (2007). Six weeks of intensive treadmill training improves gait and quality of life in patients with Parkinson's disease: a pilot study. Archives of Physical Medicine and Rehabilitation, $88(9)$,

1154-1158. http://doi.org/10.1016/j.apmr.2007.05.015

Herman, T., Giladi, N., \& Hausdorff, J. M. (2009). Treadmill training for the treatment of gait disturbances in people with Parkinson's disease: a mini-review. Journal of Neural Transmission (Vienna, Austria: 1996), 116(3), 307-318. http://doi.org/10.1007/s00702-008-0139-z

Hoehn, M. M., \& Yahr, M. D. (1967). Parkinsonism: onset, progression, and mortality. 1967. Neurology, 17(5), 427-442.

Holtzman, J. D., \& Gazzaniga, M. S. (1982). Dual task interactions due exclusively to limits in processing resources. Science (New York, N.Y.), 218(4579), 1325-1327.

Kang, H. G., \& Dingwell, J. B. (2008). Effects of walking speed, strength and range of motion on gait stability in healthy older adults. Journal of Biomechanics, 41(14), 2899-2905. http://doi.org/10.1016/j.jbiomech.2008.08.002

Kummer, A., Cardoso, F., \& Teixeira, A. L. (2010). Generalized anxiety disorder and the Hamilton Anxiety Rating Scale in Parkinson's disease. Arquivos De Neuro-Psiquiatria, 68(4), 495-501.

Matsumoto, L., Magalhães, G., Antunes, G. L., \& Torriani-Pasin, C. (2014). Efeitos do estímulo acústico rítmico na marcha de pacientes com Doença de Parkinson. Revista Neurociências, 22(3), 404-409. http://doi.org/10.4181/RNC.2014.22.03.965.6p

Mehndiratta, M., Garg, R. K., \& Pandey, S. (2011). Nonmotor symptom complex of Parkinson's disease--an under-recognized entity. The Journal of the Association of Physicians of India, 59, 302308, 313.

Morris, M. E. (2000). Movement disorders in people with Parkinson disease: a model for physical therapy. Physical Therapy, 80(6), 578-597.

Nieuwboer, A., Rochester, L., \& Jones, D. (2008). Cueing gait and gait-related mobility in patients with Parkinson's disease: developing a therapeutic method based on the International Classification of Functioning, Disability, and Health. Topics in Geriatric Rehabilitation, 24(2), 151-165.

O'Shea, S., Morris, M. E., \& Iansek, R. (2002). Dual task interference during gait in people with Parkinson disease: effects of motor versus cognitive secondary tasks. Physical Therapy, 82(9), 888-897.

Paterson, K. L., Lythgo, N. D., \& Hill, K. D. (2009). Gait variability in younger and older adult women is altered by overground walking protocol. Age and Ageing, 38(6), 745-748. http://doi.org/10.1093/ageing/afp159

Podsiadlo, D., \& Richardson, S. (1991). The timed «Up \& Gon: a test of basic functional mobility for frail elderly persons. Journal of the American Geriatrics Society, 39(2), 142-148. http://doi.org/10.1111/j.15325415.1991.tb01616.x

Rochester, L., Hetherington, V., Jones, D., Nieuwboer, A., Willems, A.-M., Kwakkel, G., \& Van Wegen, E. (2005). The Effect of External Rhythmic Cues (Auditory and Visual) on Walking During a Functional Task in Homes of People With Parkinson's Disease. Archives of Physical Medicine and Rehabilitation, 86(5), 999-1006. http://doi.org/10.1016/j.apmr.2004.10.040

Scalzo, P. L., Flores, C. R., Marques, J. R., Robini, S. C. de O., \& Teixeira, A. L. (2012). Impact of changes in balance and walking capacity on the quality of life in patients with Parkinson's disease. Arquivos de Neuro-Psiquiatria, 70(2), 119-124. 
http://doi.org/10.1590/S0004-

282X2012000200009

Seco-Calvo, J., Gago-Fernández, I., Cano-de-la-Cuerda, R., \& Fernández-de-las-Peñas, C. (2012). Efectividad de los estímulos sensoriales sobre los trastornos de la marcha en pacientes con enfermedad de Parkinson. Estudio piloto. Fisioterapia, 34(1), 4-10. http://doi.org/10.1016/j.ft.2011.07.009

Suteerawattananon, M., Morris, G. S., Etnyre, B. R., Jankovic, J., \& Protas, E. J. (2004). Effects of visual and auditory cues on gait in individuals with Parkinson's disease. Journal of the Neurological Sciences, 219(1-2), 63-69. http://doi.org/10.1016/j.jns.2003.12.007
Toole, T., Maitland, C. G., Warren, E., Hubmann, M. F., \& Panton, L. (2005). The effects of loading and unloading treadmill walking on balance, gait, fall risk, and daily function in Parkinsonism. NeuroRehabilitation, 20(4), 307-322.

Watson, M. J. (2002). Refining the Ten-metre Walking Test for Use with Neurologically Impaired People. Physiotherapy, 88(7), 386-397. http://doi.org/10.1016/S0031-9406(05)61264-3

Wu, T., \& Hallett, M. (2008). Neural correlates of dual task performance in patients with Parkinson's disease. Journal of Neurology, Neurosurgery, and Psychiatry, 79(7) http://doi.org/10.1136/jnnp.2007.126599 